\title{
Is it oncologically and clinically safe to adopt a bladder sparing approach in complete responders following neoadjuvant chemotherapy alone without proceeding to a radical cystectomy?
}

\author{
Ranjan Arianayagam ${ }^{1}$, Anand Sharma ${ }^{2}$, Nikhil Vasdev ${ }^{1,3}$ \\ ${ }^{1}$ Department of Urology and Surgery, Hertfordshire and Bedfordshire Urological Cancer Centre, Lister Hospital, East and North Herts NHS Trust, \\ Stevenage, UK; ${ }^{2}$ Department of Oncology, Mount Vernon Cancer Centre, Northwood, UK; ${ }^{3}$ School of Life and Medical Sciences, University of \\ Hertfordshire, Hatfield, UK \\ Correspondence to: Mr. Nikhil Vasdev. Department of Urology, Lister Hospital (East and North Herts NHS Trust), Stevenage, SG1 4AB, UK. \\ Email: nikhilvasdev@doctors.org.uk. \\ Provenance: This is an invited Editorial commissioned by Section Editor Xiao Li (Department of Urology, Jiangsu Cancer Hospital \& Jiangsu \\ Institute of Cancer Research \& Nanjing Medical University Affiliated Cancer Hospital, Nanjing, China). \\ Comment on: Mazza P, Moran GW, Li G, et al. Conservative Management Following Clinical Complete Response to Neoadjuvant Chemotherapy of \\ Muscle Invasive Bladder Cancer: Contemporary Outcomes of a Multi-Institutional Cohort Study. J Urol 2018;200:1005-13.
}

Submitted Aug 06, 2018. Accepted for publication Aug 13, 2018.

doi: $10.21037 /$ tau.2018.08.18

View this article at: http://dx.doi.org/10.21037/tau.2018.08.18

Carcinoma of the bladder is the second most common urological cancer and $25 \%$ of the cases are muscle invasive in nature $(1,2)$. In order to get better outcomes in patients with muscle invasive bladder cancer (MIBC), neoadjuvant chemotherapy (NAC) based on platinum based regimes is used. Benefits of NAC are to lower the burden of micrometastatic disease, to investigate the chemosensitivity of the primary tumour, and also the enhanced likely tolerability of chemotherapy pre surgery (3-5). Radical cystectomy (RC) and bilateral pelvic lymphadenectomy has demonstrated to be the most appropriate treatment for MIBC $(6,7)$. Pathological stage and lymph node status are good prognostic indicators of long term clinical outcomes following surgery (8). Five-year survival in patients with MIBC ranges from $32 \%$ in lymph node positive disease to $75 \%$ in node negative disease $(9,10)$.

We congratulate the authors on producing this important paper that addresses the controversial role of a bladder sparing approach in complete responders to NAC. The rationale for a bladder sparing approach, using a combination of NAC and radical trans-urethral resection of bladder tumour (TURBT), is clear. The complexity of $\mathrm{RC}$ can result in significant patient morbidity and mortality, in addition to acceptance of a form of urinary diversion. In addition, the authors cite the results of the SWOG trial, where patients with $\mathrm{T} 0$ on final pathology had significantly improved overall survival compared to patients with residual disease, suggesting that these patients make up a subgroup of responders with a lower chance of recurrence (11).

The authors define complete clinical response (CCR) as the absence of tumour on post-chemotherapy TURBT, negative cytology and normal cross-sectional imaging. They note that there is conflicting evidence on whether cT0 status correlates with pT0 status. However, we have a number of concerns about a bladder sparing approach. A RC is still considered the gold standard for high-risk bladder cancer, including muscle invasive disease, highgrade non-muscle invasive disease and intra-vesical treatment failures. It provides local control by removing the bladder as a potential source for future metastatic deposits and future local recurrence. A RC is usually combined with an extended lymph node dissection, which provides both prognostic information and curative effect.

T0 patients are at risk of being under staged. The authors state that they used cross sectional imaging as their main technique for detection of nodal spread and systemic disease, combined with a rigorous follow up schedule. However, CT may not be accurate enough to detect all pathological nodal disease. Local staging may be more accurate with a combination of MRI with cystoscopy, rather 
than with cystoscopy, cytology and 'normal' follow-up imaging. This would be easier to implement in a prospective study as it is unlikely there would have been uniformity of follow up imaging in this retrospective study. PET CT may be a more accurate modality for occult metastatic nodal disease than CT or MRI staging alone (12).

In the study $48 \%$ of patients experienced an intra-vesical recurrence at a median of 21 months. Of these, 16 of 71 patients recurred with muscle invasive disease. In our view, this is an unacceptably high risk of recurrence, particularly in a cohort with predominantly low to moderate volume solitary T2 lesions with only $29 \%$ having concomitant CIS. The median time to recurrence was 5.4 months, which suggests a short disease-free period. However, in those patients who accepted salvage RC, it was successful in $85 \%$ of patients with an intra-vesical recurrence and the 5-year overall survival for the study cohort was $86 \%$.

The success of local treatment may also be in part dependent on the radical nature of the TURBTs carried out prior to NAC. The authors note that almost $90 \%$ had more than 1 TURBT prior to NAC, which suggests that cT0 status may be related to a high quality, radical TURBT, rather than just to NAC.

A retrospective study that considered the risk of lymph node recurrence in $\mathrm{T} 0$ disease concluded that patients could be stratified into 2 groups of responders depending on their initial pathology. Those with advanced (T4 or node positive) disease had a significantly higher risk of lymph node recurrence despite clinical T0 disease after NAC (13). As they suggest, local, nodal or systemic under staging may serve as a potential source of disease recurrence. Whilst not applicable to all T0 patients, this reinforces a major concern with the bladder sparing approach. The authors suggest that because the 4 patients who died from extra-vesical recurrences did not have pelvic nodal disease, they would not have benefited from cystectomy. Again, however, in our view, this discounts the potential role of the intact bladder as a potential source of systemic disease.

We are concerned about the possibility of micrometastatic disease and suggest that this may be due to a lack of local control with bladder sparing approaches. On the other hand, this is a different model of disease control to chemo-radiotherapy, where radiotherapy achieves a measure of local control.

The authors suggest careful selection of T0 patients is imperative, and that patients with hydronephrosis and higher stage disease should be excluded from bladder conservation. Lower clinical stage has also been confirmed as a predictor of $\mathrm{pT} 0$ disease in the literature (14).

This paper aims to provide a possible rationale for a bladder sparing approach in carefully selected patients with lower risk $\mathrm{T} 2$ disease who have a good response to NAC and TURBT. However, we have concerns about its application even in this relatively lower risk cohort. This includes the high recurrence rate, the time to recurrence, the methods used to class patients as cT0, and the possibility of the bladder remaining as a source of systemic disease. The low proportion of cT3 disease (1\%), likely explains for the low event rate observed in this study, contributing to the inadequate power of the study to answer the primary study question. In 2006, Yu et al. published a series of 311 patients who had pT2 disease, with a 10 -year recurrence free and overall survival rates of $72 \%$ and $47 \%$ respectively. This study also showed a higher risk of node positive disease in pT2b vs. pT2a tumors (30\% vs. $14 \%, \mathrm{P}<0.001)(7)$. Mazza et al. have failed to show the distribution of the patients based on the pT2 status (15). This would have implications in the prognosis as $\mathrm{pT} 2 \mathrm{~b}$ tumors have high risk of node positive disease, which in turn also affects the prognosis (16).

Undoubtedly, several questions remain. What is the role of chemotherapy and surgery in pT2 MIBC? What parameters define a patient at high risk of recurrence? Are there any biomarkers, which can identify patients with high risk of recurrence? Is there any role for immunotherapy drugs in these patients? Although this paper addresses the question of conservative management of MIBC, the small sample size, lack of randomisation, retrospective study and non-inclusion of patients with high-risk features (pT3, node positive), makes it imperative to conduct further studies, which can address these questions, possible in an adequately powered prospective trial.

In our view, these concerns would preclude us from offering a bladder sparing approach to patients with $\mathrm{T} 2$ disease. The author's conclusions require a prospective randomized controlled trial comparing bladder sparing, after NAC, with $\mathrm{RC}$ in patients who have refused $\mathrm{RC}$ as a treatment option.

With the advent of novel targeted treatments and use of immune checkpoint inhibitors have a significant as yet unexplored potential in the NAC risk reducing setting. The development of biomarkers and improved gene profiling of tumors (17), appropriate personalised treatment will be the future in management of MIBC.

\section{Acknowledgements}

None. 


\section{Footnote}

Conflicts of Interest: The authors have no conflicts of interest to declare.

\section{References}

1. Antoni S, Ferlay J, Soerjomataram I, et al. Bladder Cancer Incidence and Mortality: A Global Overview and Recent Trends. Eur Urol 2017;71:96-108.

2. Babjuk M, Oosterlinck W, Sylvester R, et al. EAU guidelines on non-muscle-invasive urothelial carcinoma of the bladder, the 2011 update. Eur Urol 2011;59:997-1008.

3. Alfred Witjes J, Lebret T, Comperat EM, et al. Updated 2016 EAU Guidelines on Muscle-invasive and Metastatic Bladder Cancer. Eur Urol 2017;71:462-75.

4. Sternberg CN, Bellmunt J, Sonpavde G, et al. ICUDEAU International Consultation on Bladder Cancer 2012: Chemotherapy for urothelial carcinoma-neoadjuvant and adjuvant settings. Eur Urol 2013;63:58-66.

5. Culp SH, Dickstein RJ, Grossman HB, et al. Refining patient selection for neoadjuvant chemotherapy before radical cystectomy. J Urol 2014;191:40-7.

6. Gupta NP, Kolla SB, Seth A, et al. Radical cystectomy for bladder cancer: A single center experience. Indian J Urol 2008;24:54-9.

7. Yu RJ, Stein JP, Cai J, et al. Superficial (pT2a) and deep (pT2b) muscle invasion in pathological staging of bladder cancer following radical cystectomy. J Urol 2006;176:4938; discussion 498-9.

8. Bassi P, Ferrante GD, Piazza N, et al. Prognostic factors of outcome after radical cystectomy for bladder cancer: a retrospective study of a homogeneous patient cohort. J Urol 1999;161:1494-7.

9. Sternberg CN, Skoneczna I, Kerst JM, et al. Immediate versus deferred chemotherapy after radical cystectomy in

Cite this article as: Arianayagam $\mathrm{R}$, Sharma A, Vasdev N. Is it oncologically and clinically safe to adopt a bladder sparing approach in complete responders following neoadjuvant chemotherapy alone without proceeding to a radical cystectomy? Transl Androl Urol 2018;7(Suppl 6):S741-S743. doi: 10.21037/tau.2018.08.18 patients with pT3-pT4 or $\mathrm{N}+\mathrm{M} 0$ urothelial carcinoma of the bladder (EORTC 30994): an intergroup, open-label, randomised phase 3 trial. Lancet Oncol 2015;16:76-86.

10. Yafi FA, Aprikian AG, Chin JL, et al. Contemporary outcomes of 2287 patients with bladder cancer who were treated with radical cystectomy: a Canadian multicentre experience. BJU Int 2011;108:539-45.

11. Grossman HB, Natale RB, Tangen CM, et al. Neoadjuvant chemotherapy plus cystectomy compared with cystectomy alone for locally advanced bladder cancer. $\mathrm{N}$ Engl J Med 2003;349:859-66.

12. Kibel AS, Dehdashti F, Katz MD, et al. Prospective study of $[18 \mathrm{~F}]$ fluorodeoxyglucose positron emission tomography/computed tomography for staging of muscle-invasive bladder carcinoma. J Clin Oncol 2009;27:4314-20.

13. Kaag MG, Milowsky MI, Dalbagni G, et al. Regional lymph node status in patients with bladder cancer found to be pathological stage $\mathrm{T} 0$ at radical cystectomy following systemic chemotherapy. BJU Int 2011;108:E272-7.

14. Zargar H, Espiritu PN, Fairey AS, et al. Multicenter assessment of neoadjuvant chemotherapy for muscleinvasive bladder cancer. Eur Urol 2015;67:241-9.

15. Mazza P, Moran GW, Li G, et al. Conservative Management Following Clinical Complete Response to Neoadjuvant Chemotherapy of Muscle Invasive Bladder Cancer: Contemporary Outcomes of a Multi-Institutional Cohort Study. J Urol 2018;200:1005-13.

16. Cheng L, Neumann RM, Scherer BG, et al. Tumor size predicts the survival of patients with pathologic stage $\mathrm{T} 2$ bladder carcinoma: a critical evaluation of the depth of muscle invasion. Cancer 1999;85:2638-47.

17. Glaser AP, Fantini D, Shilatifard A, et al. The evolving genomic landscape of urothelial carcinoma. Nat Rev Urol 2017;14:215-29. 